DR. GIAN MARCO DE MARCHIS (Orcid ID : 0000-0002-0342-9780)

Article type : Original Article

\title{
Serum Neurofilament Light Chain in Patients with Acute
}

\section{Cerebrovascular Events}

\section{Running Title: Admission SNfL associated with clinical stroke severity, but not infarct size}

Gian Marco De Marchis, MD MSc ${ }^{1} *$; Mira Katan, MD MSc ${ }^{2} *$; Christian Barro, MD ${ }^{1 *}$; Joachim Fladt, MD ${ }^{1}$; Christopher Traenka, MD ${ }^{1}$; David J Seiffge, $\mathrm{MD}^{1}$; Lisa Hert, MD $^{1}$; Henrik Gensicke, $\mathrm{MD}^{1}$; Giulio Disanto, $\mathrm{MD}^{3}$; Raoul Sutter ${ }^{1,4}$, MD; Nils Peters, MD ${ }^{1}$; Hakan Sarikaya ${ }^{5}$, MD; Barbara Goeggel-Simonetti ${ }^{5,6}$, MD; Marwan El-Koussy, $\mathrm{MD}^{7}$; Stephan Engelter, $\mathrm{MD}^{1}$; Philippe A Lyrer, $\mathrm{MD}^{1}$; Mirjam Christ-Crain, $\mathrm{MD} \mathrm{PhD}^{8}$; Marcel Arnold, $\mathrm{MD}^{5}$; Jens Kuhle, $\mathrm{MD} \mathrm{PhD}^{1 *}$, Leo H Bonati, $\mathrm{MD}^{1 *}$

*equally contributing authors

\begin{abstract}
Affiliations:
${ }^{1}$ Department of Neurology \& Stroke Center, University Hospital Basel, University of Basel, Switzerland

${ }^{2}$ Department of Neurology, University Hospital Zurich, Zurich, Switzerland

${ }^{3}$ Department of Neurology, Neurocenter of Southern Switzerland, Lugano, Switzerland

${ }^{4}$ Department of Intensive Care Medicine, University Hospital Basel, University of Basel, Switzerland

${ }^{5}$ Department of Neurology, Inselspital, Bern, Switzerland

${ }^{6}$ Department of Pediatrics, San Giovanni Hospital, Bellinzona, Switzerland

${ }^{7}$ Department of Neuroradiology, Inselspital, Bern, Switzerland

${ }^{8}$ Department of Internal Medicine and Department of Clinical Research, Division of Endocrinology, Diabetology and Metabolism, University Hospital Basel, University of Basel, Switzerland

This article has been accepted for publication and undergone full peer review but has not been through the copyediting, typesetting, pagination and proofreading process, which may lead to differences between this version and the Version of Record. Please cite this article as doi: 10.1111/ene.13554
\end{abstract}

This article is protected by copyright. All rights reserved. 


\section{ClinicalTrials.gov: NCT00878813}

\section{Corresponding author:}

Dr. Gian Marco De Marchis, MD MSc PD

Department of Neurology \& Stroke Center

University Hospital Basel

Petersgraben 4

4031 Basel, Switzerland

$e:$ gian.demarchis@usb.ch

T: +41613287302

Supplementary Information: Disclosures

\section{ABSTRACT}

Background and Purpose: Serum Neurofilaments are markers of axonal injury. We addressed their diagnostic and prognostic role in acute ischemic stroke (AIS) and TIA.

Methods: Nested within a prospective cohort study, we compared serum neurofilament light chain levels (sNfL) drawn within 24 hours from symptom onset in patients with AIS or TIA. Patients without MRI on admission were excluded. We assessed if $\mathrm{SNfL}$ are associated with: (i) clinical severity on admission, (ii) diagnosis of AIS vs TIA, (iii) infarct size on admission diffusion weighted imaging (MR-DWI), and (iv) functional outcome at 3 months.

Results: We analyzed 504 patients with AIS and 111 patients with TIA. On admission, higher NIHSS scores were associated with higher sNfL: NIHSS $<7$ (13.1 pg/ml [IQR: 5.3-27.8]), NIHSS 7-15 (IQR: $16.7 \mathrm{pg} / \mathrm{ml}$ [7.4-34.9]), NIHSS $>15$ (21.0 pg/ml [IQR: 9.3-40.4]) ( $\mathrm{P}=0.01)$. Compared to AIS, patients with TIA had lower sNfL levels $(9.0 \mathrm{pg} / \mathrm{ml}$ [95\%-CI 4.0-19.0] vs. $16.0 \mathrm{pg} / \mathrm{ml}$ [95\%-CI 7.3-34.4], $\mathrm{P}<0.001)$, also after adjusting for age and NIHSS ( $\mathrm{P}=0.006)$. Among patients with AIS, infarct size on admission MR-DWI was not associated with sNfL, neither in univariate analysis ( $\mathrm{P}=0.15)$, nor after adjusting for age and NIHSS on admission $(\mathrm{P}=0.56)$. Functional outcome three months after stroke was not associated with sNfL after adjusting for established predictors.

Conclusions: In conclusion, among patients admitted within 24 hours of AIS or TIA onset, admission sNfL were associated with clinical severity on admission and TIA diagnosis, but not with infarct size on MR-DWI acquired on admission or functional outcome at three months.

This article is protected by copyright. All rights reserved. 
Key words: Biomarker — Neurofilaments Light Chain — Stroke — TIA — MRI — Outcome

\section{INTRODUCTION}

Blood markers of acute brain injury may help in the management of patients with acute cerebrovascular events. Blood markers may help discern patients with an acute ischemic stroke (AIS) from transient ischemic attack (TIA), serve as surrogate marker of acute infarct size, and improve models predicting functional outcome.

Neurofilaments are $10 \mathrm{~nm}$ polypeptide filaments constituting a major component of the axonal cytoskeleton. Neurofilaments consist of at least three subunits: light chain (NfL), medium chain (NfM), heavy chain $(\mathrm{NfH})$ and of alpha-internexin.(1) After neuro-axonal injury, neurofilaments are released into the extracellular space, cerebrospinal fluid and peripheral blood.(1) In peripheral blood, reliable detection of the neurofilament light chain samples has become possible only in recent years.(2) Serum neurofilament light chain (sNfL) has been shown to be a marker of injury in a number of chronic neurological disorders, including multiple sclerosis(3), frontotemporal dementia(4), amyotrophic lateral sclerosis,(5) and in some acute neurological disorders such as acute spinal injury (6) and traumatic brain injury(7). However, in AIS and TIA, the role of sNfL has not yet been investigated in large study populations. In this prospective cohort study, we assessed if sNfL are associated with: (i) clinical severity on admission, (ii) diagnosis of AIS vs. TIA, (iii) infarct size on admission MR-Diffusion Weighted Imaging (MR-DWI), and (iv) functional outcome at 3 months. A control group was included.

\section{PATIENTS AND METHODS}

Ethics Statement: This study (ClinicalTrials.gov: NCT00878813) was approved by the Ethics Committees. All patients or their welfare guardians provided written informed consent for the collection of data, blood samples, and subsequent analyses.

Study Design, Inclusion and Exclusion Criteria: This is a prospective cohort study nested within the CoRisk Study, a multicenter prospective cohort study described previously (CoRisk-Study, ClinicalTrials.gov: NCT00878813).(8) In the CoRisk study, we included consecutive patients $>18$ years with AIS or TIA within 24 hours of symptom onset, admitted to the emergency department of the University Hospital Bern, Basel and Frankfurt a. M. between March 24, 2009 and April 8, 2011.

Inclusion and Exclusion Criteria: For this nested cohort study, we selected patients admitted to the University Hospital Bern, which represent the majority of the CoRisk cohort (934/1085, 86\%), with a diagnosis of AIS or TIA. AIS was defined as neurological dysfunction lasting $>24$ hours with an acute

This article is protected by copyright. All rights reserved. 
lesion on MR-DWI and no acute intracranial bleeding. TIA was defined as neurological dysfunction lasting $\leq 24$ hours and caused by focal brain ischemia without an acute lesion on MR-DWI (a modified tissue-based definition).(9) To discriminate AIS from TIA, and to reliably assess the size of the acute infarct and sNfL, we excluded patients without Magnetic Resonance Imaging (MRI) on admission day $(n=270)$. Finally, we excluded patients with symptoms lasting $>24$ hours but no acute infarct on MR-DWI, as they could neither be classified as AIS nor TIA $(n=39)$, as well as patients with insufficient serum left to measure $\mathrm{SNfL}(\mathrm{n}=10)$.

The control group consisted of volunteers aged between 18-70 years, without any known neurological disease. Information on cardiovascular risk factors was not available for the control group.

Size Estimation of Acute Infarct: All MRI were acquired prior to thrombolysis, if thrombolysis was performed. Infarct sizes on MR-DWI were estimated by consensus of two experienced raters unaware of the clinical and laboratory findings. Infarct size was estimated by a commonly used semiquantitative method validated for acute infarcts.(10) Infarct sizes were categorized into: i) small infarct with a volume of $<10 \mathrm{~cm}^{3}$, ii) medium infarct of $10-100 \mathrm{~cm}^{3}$, iii) large infarct with a volume of more than $100 \mathrm{~cm}^{3}$.

Functional Outcome: Trained stroke physicians and study nurses assessed functional outcome three months after the acute stroke, either during an outpatient visit or with a structured follow-up telephone interview. Unfavorable functional outcome was defined as a modified Rankin Scale (mRS) score of 3 to $6 .(11)$

Biomarker Measurement: Blood was drawn in the emergency room upon admission (day 0). To study the temporal evolution of sNfL levels, a subset of patients who underwent thrombolysis received a second blood sampling after 24 hours (day 1). After centrifugation for 20 minutes at 3 '000g at room temperature, serum (from EDTA tube) was aliquoted. Tubes were frozen locally at $70^{\circ} \mathrm{C}$. sNfL concentrations were measured in duplicate by a previously described and slightly modified electrochemiluminescence (ECL) immunoassay.(2) For further detail please see the supplementary information.

Statistics: Discrete variables were expressed as counts (percentages) and continuous variables as medians (interquartile range [IQR]). The distribution of raw sNfL levels was skewed. After log transformation with base of 10 of sNfL levels, the distribution of raw sNfL levels approximated a normal distribution. To compare variables, we used the Fisher's exact test (for categorical data), the Mann-Whitney-U test (for unmatched continuous data) and the Snedecor-Cochran test (for matched continuous data, i.e. sNfL levels of day 0 to day 1). To assess the independent association of sNfL with dichotomous endpoint (i.e. AIS vs. TIA), we computed multivariate logistic regression models adjusted for established predictors.

Ordinal regression models were used to explore the association between SNfL and ordinal endpoints (i.e. NIHSS-categories and infarct size). Linear regression models were used to explore the association between two continuous variables (e.g., $\log (\mathrm{sNfL})$ and hours between symptom onset and venipuncture). We report odds ratios (OR) along with 95\% confidence intervals as measure of association and uncertainty, respectively.

Sensitivity and specificity of sNfL were calculated based on Receiver-Operating-Characteristic (ROC)-curve (C-statistics). Testing was two-sided and $P$ values $<0.05$ were considered to indicate statistical significance. Statistics were calculated using Stata Statistical Software: Release 14.2.

This article is protected by copyright. All rights reserved. 


\section{RESULTS}

We included 504 patients with AIS, 111 with TIA, and 165 volunteers in the control group. Table 1 details the baseline characteristics including sNfL levels of patients with AIS or TIA. In the control group, median age was 48 years (IQR: $38-56$ ), and 110 volunteers were women (67\%). The control group had lower sNfL levels than patients with AIS or TIA (2.5 pg/ml [IQR: 0.4-5.4] vs. $15.0 \mathrm{pg} / \mathrm{ml}$ [IQR: 6.1-31.8], $\left.\mathrm{P}_{\text {adjusted for age }}<0.001\right)$. On admission of patients with AIS or TIA, higher NIHSS scores were associated with higher sNfL NIHSS $<7$ (IQR: $13.1 \mathrm{pg} / \mathrm{ml}$ [5.3-27.8]), NIHSS 7-15 (16.7 pg/ml [IQR: 7.4-34.9]), NIHSS $>15$ (21.0 pg/ml [IQR: 9.3-40.4]) $\left(\mathrm{P}_{\text {for trend }}=0.03\right.$, adjusted for age, etiology, and time between symptom onset and venipuncture). Compared to AIS, patients with TIA had lower sNfL levels also after adjusting for age and NIHSS ( $\mathrm{P}=0.006$, Table $1 \& 2$ ). However, the association magnitude between SNfL and TIA was moderate, with an area under the curve of sNfL of 0.64 (95\% CI $0.58-0.69, \mathrm{P}<0.001$ ), indicating that $36 \%$ of patients with TIA will actually have higher sNfL than patients with AIS. Among patients with AIS, infarct size on admission MRI-DWI was not associated with sNfL, neither in univariate analysis $(\mathrm{P}=0.15)$, nor after adjusting for age and NIHSS on admission $(\mathrm{P}=0.56$, Table 2$)$.

Within the first 24 hours of symptom onset, the delay from stroke onset to venipuncture was neither associated with sNfL levels in the unadjusted analysis (R-squared=0.00, $\mathrm{P}=0.67$ ), nor in the analysis adjusted for stroke severity on admission (adj. R-squared=0.01, $\mathrm{P}=0.71$ ), with no difference across the three subgroups stratified by infarct size $\left(\mathrm{P}_{\text {for interaction }}=0.47\right)$. Among patients with AIS and TIA, sNfL were not significantly associated with leucocytes $(\mathrm{P}=0.23)$ or $\mathrm{CRP}(\mathrm{P}=0.12)$. Twenty-four hours after admission, 84 patients with AIS undergoing thrombolysis had a second venipuncture to assess SNfL. Before thrombolysis, median sNfL levels were $13.7 \mathrm{pg} / \mathrm{ml}$ (IQR: 7.0-27.2), 24 hours later $17.1 \mathrm{pg} / \mathrm{ml}$ (IQR: 9.6-35.3, $\mathrm{P}=0.06$ ). A statistically significant increase in sNfL from the admission day to the day after was seen only in the subgroup with a large infarct size on admission $(n=16)$, whereby sNfL increased from $12.9 \mathrm{pg} / \mathrm{ml}$ (IQR: 6.9-32.6) to $38.6 \mathrm{pg} / \mathrm{ml}$ (IQR: 20.1-112.5, P=0.02) (Figure 2). Any increase in sNfL levels from day 0 to $1(\mathrm{n}=51,61 \%)$ had a sensitivity of $81 \%$ and a specificity of $44 \%$ for a large infarct size (Area under the Curve: 0.78, 95\%-CI: 0.63-0.92). After adjusting for age and NIHSS, patients with the greatest increase in SNfL from day 0 to day $1\left(4^{\text {th }}\right.$ vs. $1^{\text {st }}$ quartile of increase $)$ had an almost five-fold higher odds of having a larger infarct size on admission MR-DWI (OR=4.72, [95\%-CI: 1.10-20.30], P=0.04).

At 3 months, follow-up information was available for 487 out of the 504 patients classified as AIS (follow-up rate: 97\%). A poor functional outcome was observed in 153 patients (30\%). Their baseline sNfL levels were more than twice as high as in patients with good functional outcome $(26 \mathrm{pg} / \mathrm{ml}$ [IQR: 13-47] vs. 13 pg/ml [IQR: 6-30], P<0.001). After adjusting for age, NIHSS, and the infarct size on MR-DWI, sNfL were not significantly associated with functional outcome at 3 months (Table 2).

This article is protected by copyright. All rights reserved. 


\section{DISCUSSION}

In this cohort study, patients with AIS or TIA had higher sNfL levels than volunteers without neurological diseases. Clinical severity on admission was associated with sNfL levels drawn in the first 24 hours of stroke onset. For the diagnosis of TIA versus AIS - i.e. absence vs. presence of an infarct on MR-DWI - sNfL levels discriminated moderately. These findings support the concept that sNfL mirror axonal damage. Among patients with AIS, infarct size on MR-DWI was not significantly associated with sNfL levels, at least on blood drawn on admission. From admission day to the following one, patients with large infarcts on MR-DWI had a significant increase in sNfL levels, suggesting that sNfL levels peak only after several days. Three-month outcome was not associated, after adjusting for established predictors, with sNfL levels drawn on admission.

Like our study, two prior studies compared patients with AIS to healthy controls, and found higher blood Nf levels among AIS patients.(12,13) Among patients with AIS, two prior studies observed a significant association between clinical severity on admission and blood Nf. $(12,14)$ A study with 18 AIS patients found no significant association between NIHSS and sNf, a finding likely due statistical underpower. (13) In our study, patients with TIA had 1.7-fold lower sNfL compared to patients with AIS, while a study on patients with non-traumatic cervical artery dissection found 6.6-fold lower sNfL compared to patients with AIS. In the latter study, sNfL were assessed within 30 days from symptom onset (14), i.e. later than in our study, in which sNfL were assessed on admission in all patients.(14) During the longer time interval, more NfL are released from damaged axons into the blood stream, a phenomenon that may explain the greater difference between TIA in AIS seen when sNfL are assessed in blood drawn up to 30 days from symptom onset.

In our study, the lack of association between infarct size on MR-DWI and sNfL levels on admission apparently contrasts with a previous cohort study with AIS patients, where sNfH levels did correlate with infarct size on MRI, available in 17 patients.(12) However, in our study, the median time interval between AIS onset and the first blood sample ( 2.8 hours) was shorter than in the cited study ( 2.8 hours vs. 7-10 days after stroke onset).(12) Again, this observation suggests a delay in the increase of sNf levels after AIS, weakening the association between infarct size and sNfL assessed in blood drawn as early as 24 hours of symptom onset. Moreover, it is possible that sNfL levels rise faster in patients with large, rather small, infarcts. In support of this view, we found a significant sNfL increase from day 0 over day 1 only among patients with large infarcts. While we did not have longitudinal blood samples after day 1 , sNfH reached their peak at week 3 in $80 \%$ of the AIS patients in the study by Singh et al.(12) Similarly, a significant increase in sNfL over the first 30 days was observed among AIS patients due to non-traumatic vertebral or carotid artery dissection.(14) In contrast, over the first 6 days of AIS, no significant sNfH increase was observed among 16 patients with AIS, although statistical power was likely too low to detect an increase in sNfH over time.(13) Among 27 patients with acute spinal injury, sNfL increased steadily over the first 7 days.(6) Overall, these findings point to a delayed increase of sNfL in serum after AIS or TIA, making sNf unviable as acute ischemic markers in the emergency setting despite their alluring pathophysiological pathway. In contrast, sNfL seem to be a promising marker for chronic diseases of the central nervous system, like multiple sclerosis (15), amyotrophic lateral sclerosis (16), and frontotemporal dementia (4). For instance, in multiple sclerosis, sNfL are associated with gadolinium-enhancing lesions, Expanded Disability Status Scale assessments, risk of relapses and disability progression.(3)

This article is protected by copyright. All rights reserved. 
Among AIS patients with poor functional outcome at 3 months, we found higher sNfL on admission, but the association was not significant after correction for age, NIHSS and infarct size, similarly to the previous study on patients with cervical artery dissection.(14) Another study found that poor functional outcome at 6 months was associated with higher sNfH levels drawn 3 weeks after AIS, but confounders were not adjusted for.(12) Thus, sNfL do not seem to add prognostic information beyond established predictors like NIHSS and infarct size. In contrast, two prior prospective cohort studies showed that copeptin levels measured on blood drawn on admission reclassify $11.8 \%$ of AIS-patients for the prediction of 3-month disability and $37.2 \%$ for the prediction of 3-month mortality. The reason for the greater prognostic performance of copeptin over sNf may be the faster release of copeptin into the blood stream, given that copeptin does not need to cross the blood-brain barrier, since it secreted into the blood stream from the posterior pituitary gland. (17) (18)

The strengths of this work are the large sample size, the inclusion of patients with symptom onset within 24 hours along with discrimination of AIS from TIA based on MR-DWI acquired on admission. The present study has limitations. First, the number of longitudinal samples in time was too small to reliably assess the dynamic of sNfL, and only patients treated with thrombolysis received a second venipuncture for sNfL. Thrombolysis may have mitigated axonal damage, and, consequently, the rise of sNfL over the first 24 hours since admission. We aimed, however, to primarily assess sNfL as blood marker in the emergency situation. Second, measurement of infarct size was semi-quantitative, although based on a published and validated method. However, even the discrimination ability of sNfL between TIA and AIS - i.e. absence vs. presence of an infarct on MRDWI - was moderate, questioning the clinical relevance of sNfL drawn within 24 hours of symptom onset. Third, to quantify sNfL, the ECL assay was used instead of the newly developed singlemolecule array (Simoa) method. For serum measurements, however, sNfL were reported to be highly correlated between the ECL assay and Simoa (Spearman correlation coefficient=0.86, p<0.001).(2)

In conclusion, among patients admitted within 24 hours of AIS or TIA onset, sNfL were associated with clinical severity on admission, but not with infarct size on MR-DWI acquired on admission or functional outcome at three months. The delayed increase of sNfL levels likely makes sNfL unviable as acute ischemic marker, at least when measured in blood drawn as early as 24 hours of symptom onset. Given the growing interest in the clinical role of sNfL in many neurological diseases, these findings bring new patient-oriented evidence on the role of sNfL among patients admitted with acute cerebrovascular events.

This article is protected by copyright. All rights reserved. 


\section{REFERENCES}

1. Gaiottino J, Norgren N, Dobson R, Topping J, Nissim A, Malaspina A, et al. Increased neurofilament light chain blood levels in neurodegenerative neurological diseases. PloS one. 2013;8(9):e75091.

2. Kuhle J, Barro C, Andreasson U, Derfuss T, Lindberg R, Sandelius A, et al. Comparison of three analytical platforms for quantification of the neurofilament light chain in blood samples: ELISA, electrochemiluminescence immunoassay and Simoa. Clin Chem Lab Med. 2016;54(10):1655-61.

3. Disanto G, Barro C, Benkert P, Naegelin Y, Schadelin S, Giardiello A, et al. Serum Neurofilament light: A biomarker of neuronal damage in multiple sclerosis. Ann Neurol. 2017;81(6):857-70.

4. Rohrer JD, Woollacott IO, Dick KM, Brotherhood E, Gordon E, Fellows A, et al. Serum neurofilament light chain protein is a measure of disease intensity in frontotemporal dementia. Neurology. 2016;87(13):1329-36.

5. Lu CH, Petzold A, Topping J, Allen K, Macdonald-Wallis C, Clarke J, et al. Plasma neurofilament heavy chain levels and disease progression in amyotrophic lateral sclerosis: insights from a longitudinal study. Journal of neurology, neurosurgery, and psychiatry. 2015;86(5):565-73.

6. Kuhle J, Gaiottino J, Leppert D, Petzold A, Bestwick JP, Malaspina A, et al. Serum neurofilament light chain is a biomarker of human spinal cord injury severity and outcome. Journal of neurology, neurosurgery, and psychiatry. 2015;86(3):273-9.

7. Shahim P, Gren M, Liman V, Andreasson U, Norgren N, Tegner Y, et al. Serum neurofilament light protein predicts clinical outcome in traumatic brain injury. Sci Rep. 2016;6:36791.

This article is protected by copyright. All rights reserved. 
8. De Marchis GM, Katan M, Weck A, Brekenfeld C, Mattle HP, Buhl D, et al. Copeptin and risk stratification in patients with ischemic stroke and transient ischemic attack: the CoRisk study. International journal of stroke : official journal of the International Stroke Society. 2013;8(3):214-8.

9. Easton JD, Saver JL, Albers GW, Alberts MJ, Chaturvedi S, Feldmann E, et al. Definition and evaluation of transient ischemic attack: a scientific statement for healthcare professionals from the American Heart Association/American Stroke Association Stroke Council; Council on Cardiovascular Surgery and Anesthesia; Council on Cardiovascular Radiology and Intervention; Council on Cardiovascular Nursing; and the Interdisciplinary Council on Peripheral Vascular Disease. Stroke. 2009;40(6):2276-93.

10. Sims JR, Gharai LR, Schaefer PW, Vangel M, Rosenthal ES, Lev $M H$, et al. $A B C / 2$ for rapid clinical estimate of infarct, perfusion, and mismatch volumes. Neurology. 2009;72(24):2104-10.

11. van Swieten JC, Koudstaal PJ, Visser MC, Schouten HJ, van Gijn J. Interobserver agreement for the assessment of handicap in stroke patients. Stroke. 1988;19(5):604-7.

12. Singh P, Yan J, Hull R, Read S, O'Sullivan J, Henderson RD, et al. Levels of phosphorylated axonal neurofilament subunit $\mathrm{H}(\mathrm{pNfH})$ are increased in acute ischemic stroke. Journal of the neurological sciences. 2011;304(1-2):117-21.

13. Sellner J, Patel A, Dassan P, Brown MM, Petzold A. Hyperacute detection of neurofilament heavy chain in serum following stroke: a transient sign. Neurochem Res. 2011;36(12):2287-91.

14. Traenka C, Disanto G, Seiffge DJ, Gensicke H, Hert L, Grond-Ginsbach C, et al. Serum Neurofilament Light Chain Levels Are Associated with Clinical Characteristics and Outcome in Patients with Cervical Artery Dissection. Cerebrovascular diseases. 2015;40(5-6):222-7.

This article is protected by copyright. All rights reserved. 
15. Kuhle J, Nourbakhsh B, Grant D, Morant S, Barro C, Yaldizli O, et al. Serum neurofilament is associated with progression of brain atrophy and disability in early MS. Neurology. 2017;88(9):826-

31.

16. Steinacker P, Huss A, Mayer B, Grehl T, Grosskreutz J, Borck G, et al. Diagnostic and prognostic significance of neurofilament light chain NF-L, but not progranulin and S100B, in the course of amyotrophic lateral sclerosis: Data from the German MND-net. Amyotroph Lateral Scler Frontotemporal Degener. 2017;18(1-2):112-9.

17. Katan M, Fluri F, Morgenthaler NG, Schuetz P, Zweifel C, Bingisser R, et al. Copeptin: a novel, independent prognostic marker in patients with ischemic stroke. Annals of neurology. 2009;66(6):799-808.

18. De Marchis GM, Katan M, Weck A, Fluri F, Foerch C, Findling O, et al. Copeptin adds prognostic information after ischemic stroke: results from the CoRisk study. Neurology.

2013;80(14):1278-86.

This article is protected by copyright. All rights reserved. 


\section{LEGEND TO THE TABLES AND FIGURES}

Table 1: Baseline characteristics

Table 2: Association between sNfL and Diagnosis, Clinical Severity, Infarct Sizes on MR-DWI and Functional Outcome

Figure 1: Serum Neurofilament Light Chain levels on admission of patients with acute ischemic stroke are stratified by infarct size on MR-DWI $(n=504)$. The increase in sNfL through infarct size groups was not significant.

Figure 2: Serum Neurofilament Light Chain (sNfL) levels of patients with acute ischemic stroke with a large infarct. Change (paired) from the admission day (day 0 ) to 24 hours later (day 1).

This article is protected by copyright. All rights reserved. 


\begin{tabular}{|c|c|c|c|c|c|}
\hline & $\begin{array}{l}\text { Acute Is } \\
\text { Stroke } \\
(n=\mathbf{5 0 4}\end{array}$ & chemic & $\begin{array}{l}\text { TIA } \\
(n=111)\end{array}$ & & $P$ Value \\
\hline \multicolumn{6}{|l|}{ Demographic data } \\
\hline Age, median (IQR), y & 68 & $(59-77)$ & 66 & $(56-75)$ & 0.04 \\
\hline Women, $n(\%)$ & 183 & $(36)$ & 36 & $(32)$ & 0.51 \\
\hline \multicolumn{6}{|c|}{ Laboratory values, median (IQR) } \\
\hline Serum NfL $(\mathrm{pg} / \mathrm{ml})$ & 16 & $(7-34)$ & 9 & $(4-19)$ & $<0.001$ \\
\hline Time to blood collection ( $\mathrm{min})^{*}$ & 160 & $(91-275)$ & 124 & $(80-250)$ & 0.06 \\
\hline \multicolumn{6}{|l|}{ Medical history, $n(\%)$} \\
\hline Hypertension & 322 & (64) & 75 & $(68)$ & 0.51 \\
\hline Atrial fibrillation & 71 & (14) & 8 & (7) & 0.06 \\
\hline Current smoking & 105 & (21) & 21 & (19) & 0.80 \\
\hline Diabetes mellitus & 74 & (15) & 16 & (14) & 0.54 \\
\hline Dyslipidemia & 288 & (58) & 72 & $(65)$ & 0.20 \\
\hline Previous cerebrovascular event & 79 & (16) & 15 & (14) & 0.66 \\
\hline \multicolumn{6}{|l|}{ Clinical data, median (IQR) } \\
\hline NIHSS at admission (points) & 5 & $(2-10)$ & 0 & $(0-2)$ & $<0.001$ \\
\hline NIHSS $<7, n(\%)$ & 304 & $(60)$ & 108 & $(97)$ & \multirow{3}{*}{$<0.001$} \\
\hline NIHSS $7-15, n(\%)$ & 135 & (27) & 3 & (3) & \\
\hline NIHSS $>15, n(\%)$ & 65 & (13) & 0 & $(0)$ & \\
\hline Body Mass Index $\left(\mathrm{kg} / \mathrm{m}^{2}\right)$ & 26 & $(23-28)$ & 26 & $(24-29)$ & 0.36 \\
\hline Thrombolysis, $n(\%)$ & 190 & $(38)$ & N/A & & N/A \\
\hline \multicolumn{6}{|l|}{ Infarct Volume on MR-DWI } \\
\hline None detected & 0 & 0 & 111 & $(100)$ & \multirow{4}{*}{$\mathbf{N} / \mathbf{A}^{* *}$} \\
\hline Small $\left(1-10 \mathrm{~mm}^{3}\right)$ & 244 & $(48)$ & 0 & $(0)$ & \\
\hline Medium $\left(10-100 \mathrm{~mm}^{3}\right)$ & 200 & (40) & 0 & (0) & \\
\hline Large $\left(>100 \mathrm{~mm}^{3}\right)$ & 60 & (12) & 0 & $(0)$ & \\
\hline \multicolumn{6}{|l|}{ TOAST subtype, $n(\%)$} \\
\hline Large-vessel disease & 82 & $(16)$ & 16 & (14) & \multirow{6}{*}{$<0.001$} \\
\hline Cardioembolic & 186 & (37) & 20 & (18) & \\
\hline Small-artery disease & 31 & (6) & 13 & (12) & \\
\hline Multiple causes & 45 & (9) & 13 & (12) & \\
\hline Other known & 25 & (5) & 1 & (1) & \\
\hline Undetermined & 135 & $(27)$ & 48 & $(43)$ & \\
\hline \multicolumn{6}{|c|}{$\begin{array}{l}\text { NfL denotes Neurofilament Light Chain; NIHSS: National Institute of Health Stroke Scale; mRs: } \\
\text { modified Rankin scale; N/A: not applicable } \\
\text { *time from stroke onset to venipuncture. } \\
\text { **By definition, patients with TIA had no ischemic lesion on MR-DWI (tissue based diagnosis). }\end{array}$} \\
\hline
\end{tabular}

This article is protected by copyright. All rights reserved. 


\begin{tabular}{|c|c|c|c|c|c|}
\hline \multicolumn{6}{|c|}{ Acute Ischemic Stroke \& TIA, Clinical Severity on Admission* } \\
\hline \multirow[b]{2}{*}{ Univariate OR $(95 \% \mathrm{CI})$ for $\log _{10}(\mathrm{sNfL})$} & \multirow{2}{*}{\begin{tabular}{l|l} 
OR \\
1.43
\end{tabular}} & \multicolumn{3}{|c|}{$95 \%-\mathrm{CI}$} & \multirow{2}{*}{$\begin{array}{l}P \text { Value } \\
0.002\end{array}$} \\
\hline & & 1.14 & - & 1.80 & \\
\hline Multivariate** OR $(95 \% \mathrm{CI})$ for $\log _{10}(\mathrm{sNfL})$ & 1.35 & 1.04 & - & 1.77 & 0.03 \\
\hline \multicolumn{6}{|l|}{ Acute Ischemic Stroke. Infarct Volume on MR-DWI } \\
\hline & OR & \multicolumn{3}{|c|}{$95 \%-\mathrm{CI}$} & $P$ Value \\
\hline Univariate OR $(95 \% \mathrm{CI})$ for $\log _{10}(\mathrm{sNfL})$ & 1.17 & 0.94 & - & 1.44 & 0.15 \\
\hline Multivariate ${ }^{\dagger} \mathrm{OR}(95 \% \mathrm{CI})$ for $\log _{10}(\mathrm{sNfL})$ & 1.08 & 0.84 & - & 1.39 & 0.56 \\
\hline \multicolumn{6}{|c|}{ Acute Ischemic Stroke. Unfavorable Functional Outcome at 3 Months } \\
\hline \multirow{2}{*}{ Number of Events (\% of AIS) } & \multicolumn{5}{|c|}{$153(30)$} \\
\hline & OR & \multicolumn{3}{|c|}{$95 \%-C I$} & $P$ Value \\
\hline Univariate OR $(95 \% \mathrm{CI})$ for $\log _{10}(\mathrm{sNfL})$ & 1.66 & 1.26 & - & 2.20 & $<0.001$ \\
\hline Multivariate $^{\dagger \dagger} \mathrm{OR}(95 \% \mathrm{CI})$ for $\log _{10}(\mathrm{sNfL})$ & 1.05 & 0.74 & - & 1.49 & 0.80 \\
\hline \multicolumn{6}{|l|}{ TIA vs. Acute Ischemic Stroke } \\
\hline \multirow{2}{*}{ Number of TIA (\% of overall cohort) } & \multicolumn{5}{|c|}{$111(18)$} \\
\hline & OR & \multicolumn{3}{|c|}{$95 \%-C I$} & $P$ Value \\
\hline Univariate OR $(95 \% \mathrm{CI})$ for $\log _{10}(\mathrm{sNfL})$ & 0.64 & 0.51 & - & 0.80 & $<0.001$ \\
\hline Multivariate $^{\dagger}$ OR $(95 \% \mathrm{CI})$ for $\log _{10}(\mathrm{sNfL})$ & 0.62 & 0.44 & - & 0.87 & 0.006 \\
\hline \multicolumn{6}{|c|}{$\begin{array}{l}\text { MR-DWI denotes Magnetic Resonance Diffusion-Weighted Imaging; AIS, acute ischemic stroke; OR, odds } \\
\text { ratio; CI, confidence interval; TIA, transient ischemic attack indicates odd ratio. *NIHSS-groups: 0-6 points / } 7 \\
15 \text { points / > } 15 \text { points. }\end{array}$} \\
\hline \multicolumn{6}{|c|}{ **Adjusted for age, etiology, and time between stroke onset and venipuncture } \\
\hline \multicolumn{6}{|l|}{${ }^{\dagger}$ Adjusted for age, NIHSS-group (NIHSS 0-6 / 7-15 / >16). } \\
\hline
\end{tabular}

This article is protected by copyright. All rights reserved. 


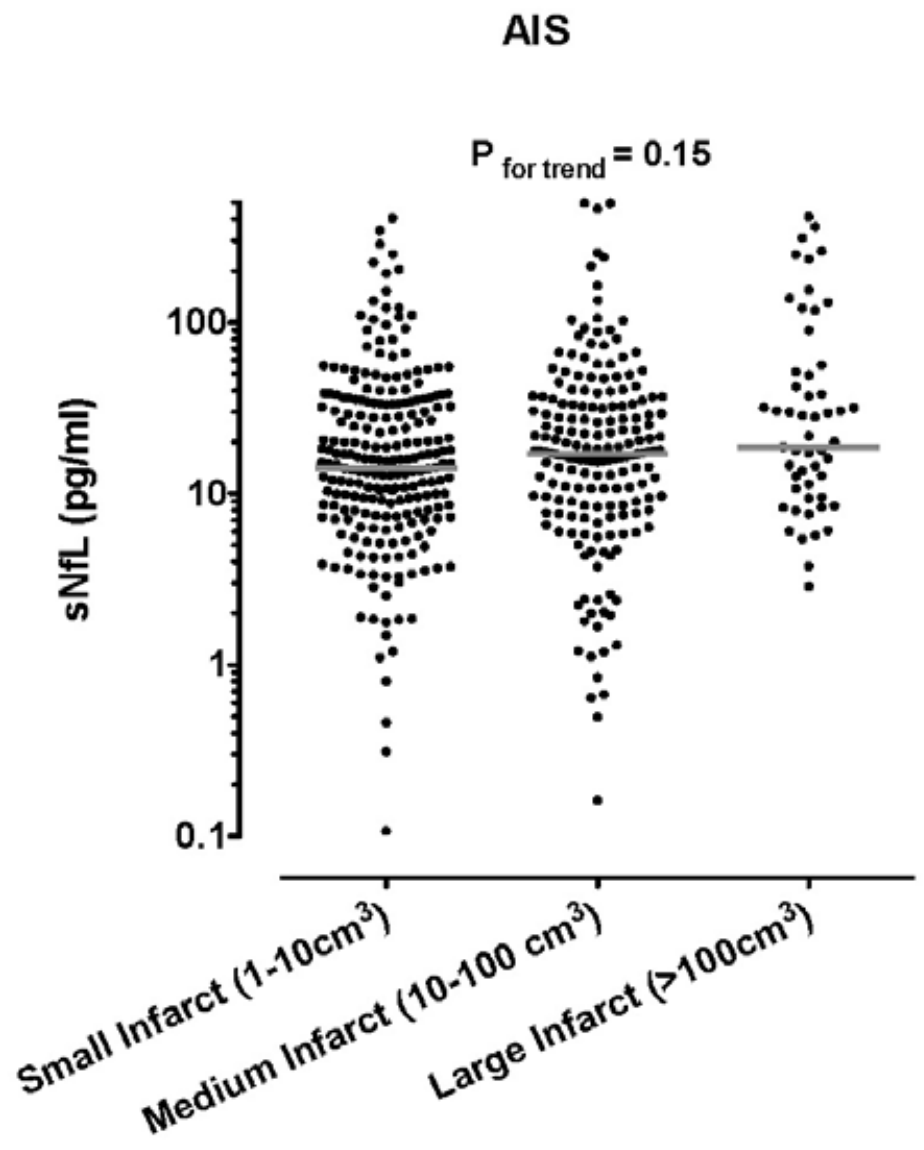

This article is protected by copyright. All rights reserved. 
AIS, Small Infarct on MR-DWI

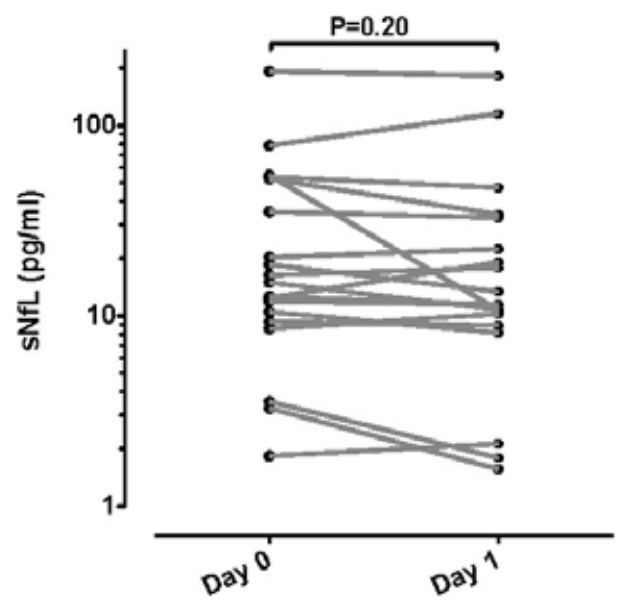

AIS, Medium Infarct on MR-DWI

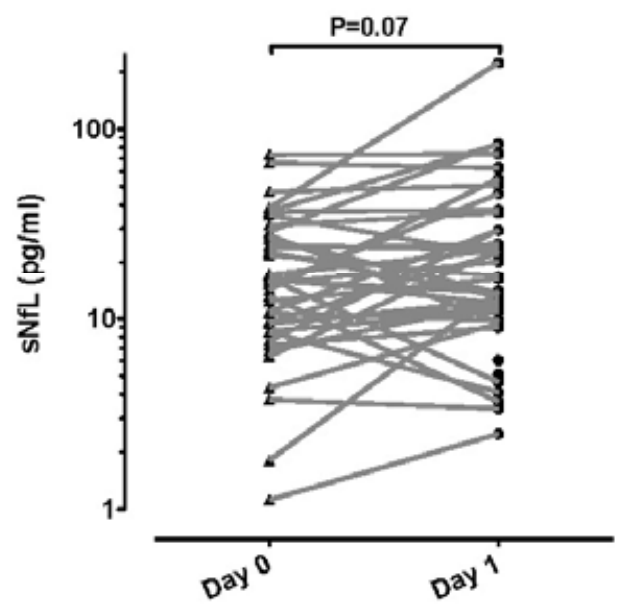

AIS, Large Infarct on Admission MR-DWI

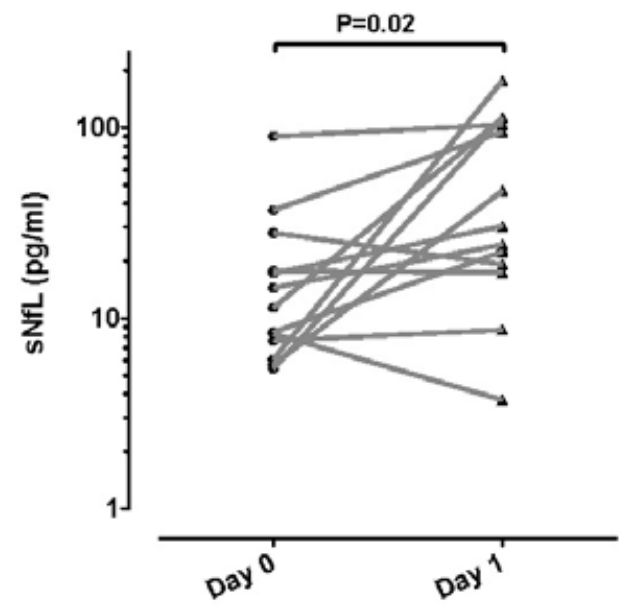

This article is protected by copyright. All rights reserved. 